\title{
Sloshing Displacement Measurements based on Morphological Image Analysis
}

\author{
E. SAATCI ${ }^{a, *}$ AND G. YAZICI ${ }^{b}$ \\ ${ }^{a}$ Department of Electrical-Electronic Eng., Istanbul Kultur University, Istanbul, Turkey \\ ${ }^{b}$ Department of Civil Eng., Istanbul Kultur University, Istanbul, Turkey
}

\begin{abstract}
Sloshing of the contained liquid is an important issue, which needs to be dealt with in the design of moving liquid storage tanks, such as fuel tanks subjected to earthquakes. Numerical methods such as the finite element method or smoothed particle hydrodynamics are commonly used to analyze the sloshing response of liquids in the design of liquid storage containers. The analysis results obtained from numerical methods are usually validated through the comparison of free surface displacements obtained from laboratory tests conducted on scaled tank models. However, the equipment required for measurement can be quite costly and the setup of the measurement system for moving containers can be a tedious process. In order to simplify the measurement setup and reduce the associated cost, we propose a measurement system based on morphological image analysis using a stationary HD camera. In order to verify the validity of the proposed method a shaking table test was conducted on a transparent prismatic liquid tank subjected to a simulated earthquake base motion. The liquid tank was filled with colored water to facilitate image analysis. The video record of the experiment was processed by the proposed algorithm implemented in the MATLAB environment to measure free surface displacements directly on the wall of the tank. The free surface displacements obtained from the video record by automated image analysis were congruent with the manual measurements conducted on the individual video frames.
\end{abstract}

DOI: 10.12693/APhysPolA.135.949

PACS/topics: sloshing, liquid storage tanks, morphological image analysis, free surface tracking, shake table

\section{Introduction}

Sloshing or the violent shaking of liquid in moving vessels is an important issue to be considered in the analysis and design of liquid storage tanks in seismically active regions. Hydrodynamic force and overturning moment generated by the movement of the contained liquid during earthquakes can cause significant damages to the tank walls and foundation. Furthermore, sloshing waves at the free liquid surface can damage the cover and result in the spilling of contents. Therefore, it is essential to have accurate estimates of hydrodynamic loads and free surface displacements due to sloshing in the seismic design of liquid storage tanks.

However, modelling of the sloshing action in moving containers is quite complex and depends on a number of factors including the tank geometry, liquid depth and the characteristics of the external excitation [1]. Various analytical models for predicting the hydrodynamic pressures and free surface wave displacements in liquid storage containers subjected to base excitation have been proposed by researchers since the early 20 th century [2-4]. The advances in the computing resources in the last few decades led to the adaptation of numerical methods such as finite element method (FEM), finite difference method (FDM), boundary element method (BEM) and smoothed particle hydrodynamics (SPH) to computational fluid dynamics (CFD) and fluid-structure interaction (FSI) problems to

*corresponding author; e-mail: e.saatci@iku.edu.tr overcome the limitations of analytical models. Extensive literature reviews of the analytical and numerical methods used in the analysis of accelerated liquid storage tanks can be obtained from Ibrahim [5], and Faltinsen and Timokha [6].

Even though analytical and numerical modelling of partially filled moving liquid containers have been studied extensively, many aspects of sloshing action are not yet fully understood. Hence, laboratory experiments are still widely needed to verify the analysis results obtained from analytical models and numerical methods. Sloshing experiments on scaled liquid containers by Luo et al. [7], Kim et al. [8], Mei-Rong et al. [9], Pistani and Thiagarajan [10], Souto-Iglesias et al. [11] and Frosina et al. [12] are among the recent examples of these studies.

An overview of these experimental studies shows that sloshing experiments are conducted on geometrically scaled liquid containers with transparent plexiglas or fiberglass walls. Hydro-elastic effects caused by the fluid structure interaction may be taken into account by adjusting the thickness of the tank walls. The liquid inside the tank may be colored to facilitate the visualization of the sloshing action. Scaled liquid containers are mounted on shake tables which are used to simulate the motions (translation, pitch and yaw) that liquid storage tanks will be subjected to in their service life.

Hydrodynamic pressures acting on the storage tank walls are recorded with pressure sensors or load cells and the deformations of the tank walls are tracked with strain gauges placed at critical locations on the tank model. Free surface waves due to sloshing may be tracked 
with capacitance wave probes placed at critical locations in the tank. Alternatively, free surface displacements can also be measured with the help of laser or ultrasonic sensors. The advantages and disadvantages of free surface and sloshing force measurement methods are discussed in [13].

Experiments are also usually recorded with one or more video cameras to view the free surface displacements throughout the experiment. Image processing of the selected frames of the video record is a commonly used method for validation purposes. Although processing an experimental image at a particular instant is quite straightforward, processing the video records of sloshing experiments manually, frame by frame, is a laborious task and there is a lack of a clearly defined procedure in the existing literature for the evaluation of the free surface displacements in sloshing experiments.

The challenges of automating the task of image processing of the frames of the video records are presented below:

- location of the tank changes in each frame since the video camera is at a fixed location. Therefore, the tank and its contents need to be identified in each frame and the image distortions due to changes in the relative distance between the tank and the video camera need to be corrected;
- small splashes do not have a profound damaging impact on the tank roof and it can be misleading to take them into account in the estimation of the freeboard requirements. Therefore, small splashes need to be filtered out during image processing;

- interference due to background objects and their reflections may make the processing of images difficult.

This paper proposes a step by step methodology to overcome the challenges delineated above in the automation of the image processing of sloshing experiment video records for tracking the free surface sloshing displacements in geometrically scaled rectangular tanks subjected to horizontal earthquake ground motions.

\section{Experimental setup and video acquisition}

The plexiglas (acrylic glass) liquid storage tank used in the experiment has a length of $400 \mathrm{~mm}$, a width of $300 \mathrm{~mm}$ and a height of $500 \mathrm{~mm}$. The plexiglas panels have a thickness of $10 \mathrm{~mm}$. The tank was filled up to a height of $100 \mathrm{~mm}$ with water which was colored red in order to improve contrast. The plexiglas panel at the back wall was covered with white paper to avoid background interference. The tank model was fixed to the shake table with 6 bolts at the base plate as shown in Fig. 1a and an accelerometer was placed on the base plate to record the acceleration time-history.

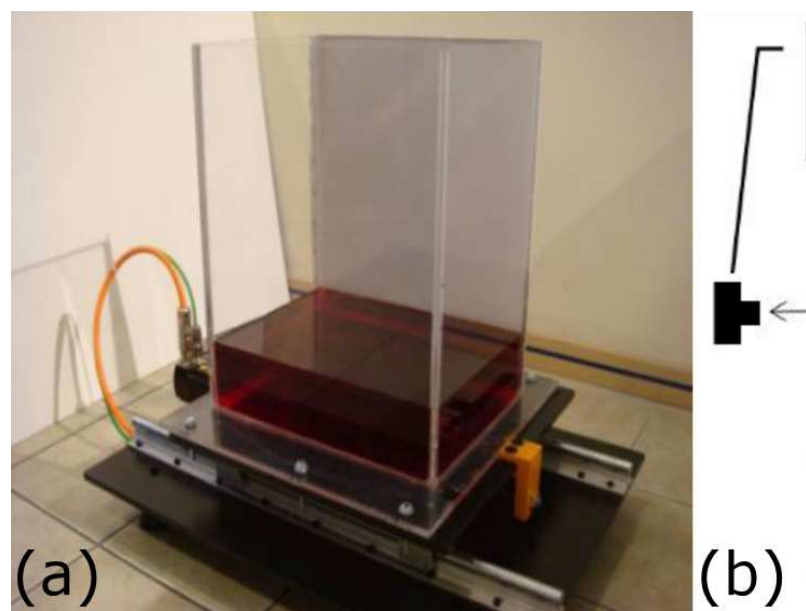

Fig. 1. (a) Experimental setup, (b) video acquisition setup.
The tabletop shake table used in the experiment has a uniaxial displacement capacity of $200 \mathrm{~mm}$. The maximum velocity which can be applied is $500 \mathrm{~mm} / \mathrm{s}$. The payload of the shake table is $50 \mathrm{~kg}$ at $2 g$ and $100 \mathrm{~kg}$ at $1 \mathrm{~g}$. The shake table has built-in functions to simulate step, cyclic (saw tooth, sinusoidal and square displacement patterns) or user defined excitations in the horizontal direction.

North-South component of the 1940 Imperial Valley Earthquake (El Centro Array 9 / I-ELC180.AT2) was used as the base excitation acceleration time-history for the experiment. Earthquake acceleration time-history record file was obtained from the PEER NGA West2 Ground Motion Database [14] and it was scaled in order to stay within the displacement, velocity and acceleration limits of the shake table, as shown in Fig. 2. Peak ground acceleration of the scaled earthquake time-history record was $0.25 \mathrm{~g}$.

Video acquisition is an important first step in the experimental measurements of liquid sloshing. Depending on the quality of the video data, results from image processing based measurements may vary. Therefore, cam- 


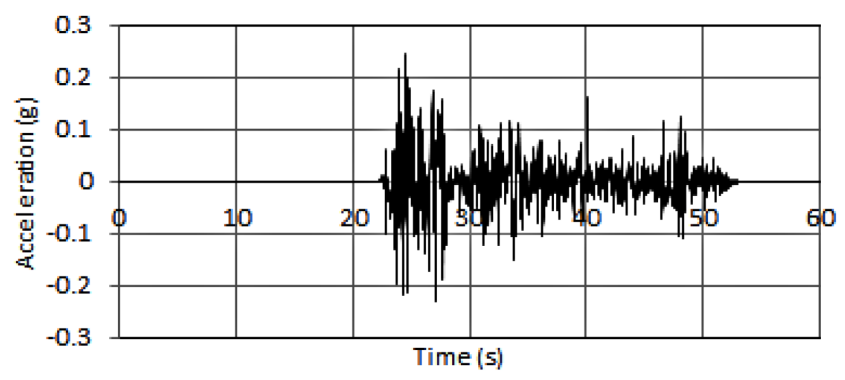

Fig. 2. Base acceleration time-history used in the experiment.

era position, installation and scene illumination are crucial to the success of the subsequent image processing in the measurements. The camera location should be chosen to minimize line-of-sight interruptions to the view of the prismatic tank. The camera should be fixed to increase the chance of stable and continuous image data acquisition.

An HD video camera (Canon Mark III $85 \mathrm{~mm} \mathrm{f/1.4)}$ was used to record movements of the colored liquid in the plexiglas prismatic tank placed on a flatbed on top of the shake table. The video camera was placed at a distance of $1.5 \mathrm{~m}$ away from the tank model and a height of $20 \mathrm{~cm}$ from the floor. The camera remained stationary throughout the experiment. White panel boards were placed on both sides of the shake table to minimize the interference of background objects during the video acquisition, as shown in Fig. 1b. Since uniaxial displacement capacity of the shake table is $20 \mathrm{~cm}$, distortions due to changes in the relative distance between the right wall of the tank and the camera are negligible in the given configuration.

\section{Image analysis for liquid sloshing experiment}

The recorded video of sloshing lasted about $20 \mathrm{~s}$ and containd 578 frames. Frames of the video were extracted and analyzed in the MATLAB environment. A sample frame from 6 th second of the video is shown in Fig. 3a.

Flowchart given in Fig. 4 shows, how the sloshing experiment video was analyzed to measure the peak value of the liquid wave at the right wall of the prismatic tank.

Before processing the frames extracted from the aforementioned video, each frame is converted from RGB to grayscale color space by using the standard NTSC conversion formula [15] which is used for calculating the effective luminance of a pixel $(i, j)$, as in Eq. (1)

$$
\begin{aligned}
& I(i, j)=0.2989 \operatorname{red}(i, j)+0.5870 \operatorname{green}(i, j) \\
& \quad+0.1140 \operatorname{blue}(i, j)
\end{aligned}
$$

In a grayscale digital image, the value of each pixel carries only the intensity information. Images of this type are composed exclusively of shades of gray, varying from black at the weakest intensity to white at the strongest. Figure $3 \mathrm{~b}$ shows grayscale converted version of the frame shown in Fig. 3a.
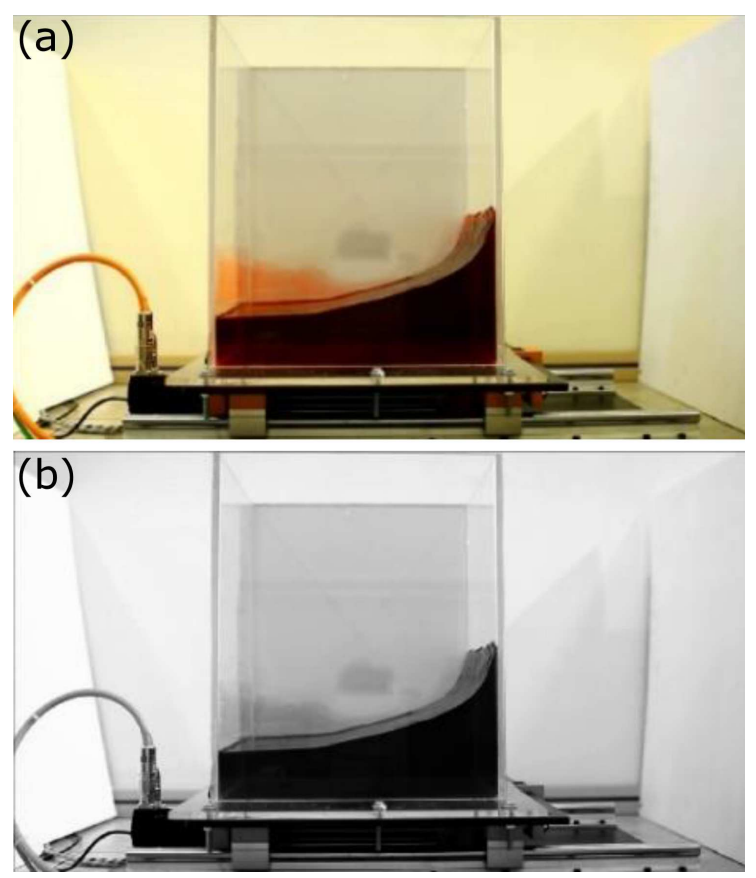

Fig. 3. (a) A sample frame from 6th second of the video, (b) grayscale converted version of the frame in (a).

To ensure successful identification of the position of the air-liquid interface on the tank side, equipment such as the shake table and flatbed on top of the shake table should be removed from the image. Therefore, after grayscale conversion, the image was cropped to remove the part lying beneath the flatbed of the shake table. Removed portion is not important as it does not carry any useful information about the problem at hand.

Digital images contain a variety of types of noise. Noise is introduced during the image acquisition process, depending on how the image is created. Because of this noise, pixel values do not reflect the true intensities of the real scene. In our case, image is acquired directly in a digital format, the CCD detector mechanism for acquiring the image can introduce noise. Therefore, after cropping, a $5 \times 5$ median filter was applied to the image for noise removal. The median filter replaces the value of the pixel of interest with the median value of the neighboring pixels and is effective in reducing irregular noise [16].

Since a colored liquid is used in the experiment, a simple thresholding operation [16] turns the image into a binary image, leaving nothing but the liquid. In order to achieve this goal, an optimal threshold value should be determined to identify colored liquid. Thresholding is the simplest method of image segmentation. The simplest thresholding method replaces each pixel in an image with a black pixel if the image intensity $I(i, j)$ at pixel $(i$, $j$ ) is less than some fixed constant $T$ (that is, $I(i, j)<T$ ), or a white pixel if the image intensity is greater than or equal to that constant. Figure 5 shows the binary image obtained after the thresholding has been performed. 


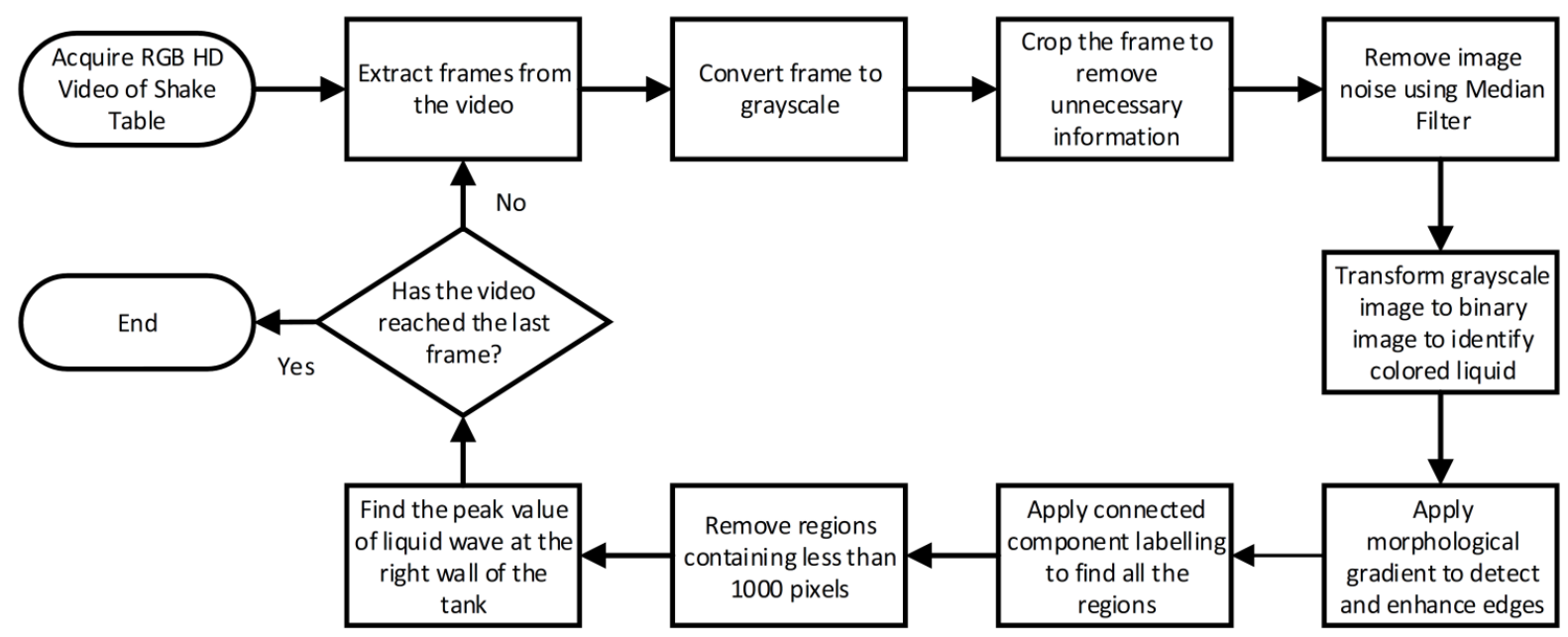

Fig. 4. Proposed algorithm.

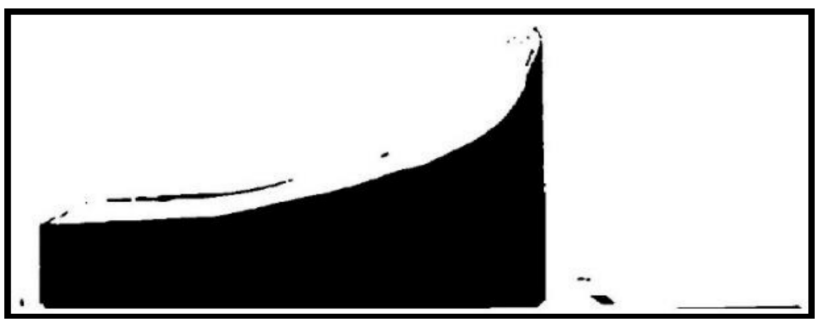

Fig. 5. Binary image obtained after the thresholding.

After grayscale to binary image conversion, air-liquid interface may be identified by detecting edges of the image. However, edge detection alone is insufficient for airliquid interface identification. There are still noises to be removed in the form of small regions on the wall of the tank resulting from splashed liquid droplets. Morphological operations, such as connected component labeling $[17,18]$ and small object removal should be applied to remove these liquid droplets after edge detection.

Edge detection [16] is an image processing technique for finding the boundaries of objects within images. It is used for image segmentation and works by detecting discontinuities in image brightness. Common traditional edge detection techniques include the Roberts, Prewitt, Sobel, Laplacian operators and the Canny edge detection. In this work, instead of traditional edge detection techniques, morphological gradient method [16, 19] was employed since morphology based edge detection perform comparably and in some cases better than conventional schemes based only on linear gradients/filters; further, the morphological gradients are computationally more efficient [20].

Dilation and erosion [16] operations are both forms of basic morphological processing; the former is used to thicken the objects, whereas the latter is used to shrink the objects. Both dilation and erosion operations are produced by the interaction of a set called a structuring element with a set of pixels of interest in the image. The structuring element has both a shape and an origin. Erosion of an image $I$ by a structuring element $B$, denoted $I \Theta B$, is defined as in Eq. (2)

$$
I \Theta B=\left\{z \mid(B)_{z} \subseteq I\right\}
$$

where $(B)_{z}$ is the shifted version of $B$ by $z$. Erosion is the set of all points $\mathrm{z}$ such that $B$, shifted by $z$, is contained by $I$. Similarly, dilation of an image $I$ by a structuring element $B$, denoted $I \oplus B$, is defined as in Eq. (3)

$$
I \oplus B=\left\{z \mid(\widehat{B})_{z} \cap I \neq \emptyset\right\}
$$

where $(\widehat{B})_{z}$ is the reflection of $B$ about its origin and followed by a shift by $z$. Dilation is the set of all shifts $z$, the shifted $\widehat{B}$ and $I$ overlap by at least one element.

Dilation and erosion can be combined with image subtraction to obtain the morphological gradient of an image [16], denoted by $G$, as in Eq. (4)

$$
G=(I \oplus B)-(I \Theta B)
$$

Morphological gradient is the difference between the dilation and the erosion operations and it enhances the edges, while suppressing the contribution of the homogeneous areas, thus producing a "derivative-like" effect [16]. Therefore, it is useful for finding the edges of an object. After using a $3 \times 3$ cross-shaped structuring element $B$, edges can be obtained as in the Fig. 6a.

In a binary image with pixel values 0 and 1 , in order for two pixels to be connected, they must be neighbors having the same value. A connected component is a set of pixels in a binary image that are connected with respect to a neighborhood rule (4-, 8- or $m$-neighborhood). Connected component labeling basically finds the connected components of a binary image. A label is a positive integer that uniquely names a connected component. In the labeling process, all the pixels in a connected component are given the same positive integer number starting from 1. Thus, all the pixels in the first connected component is labeled as 1 and those in the second as 2 and so on. 


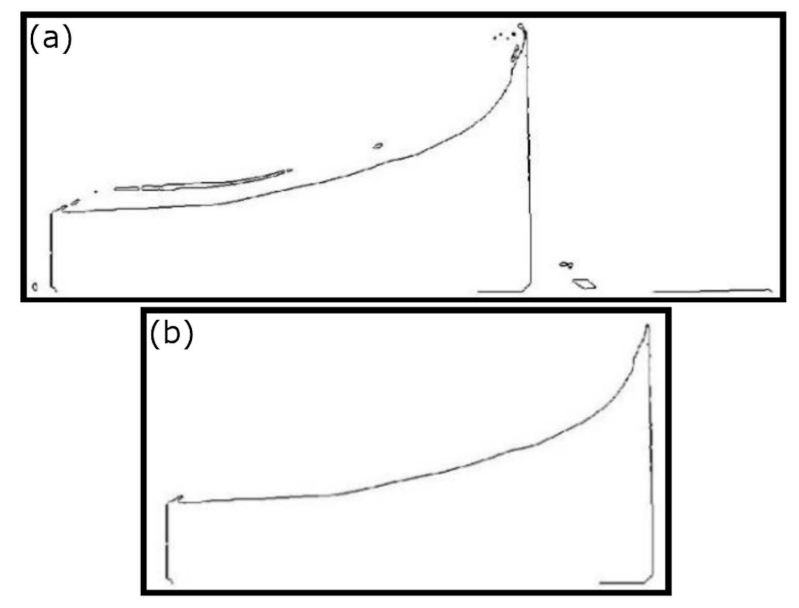

Fig. 6. (a) Morphological gradient edge detection, (b) elimination of unwanted regions by small object removal.

Small object removal process removes all connected components (objects) that have fewer pixels than a specified number of pixels from the binary image. This process is important when determining boundaries of the liquid. If the pixel regions with a small area are not removed, resulting image may contain false regions resulting from splashed liquid droplets. Unwanted regions that are visible in Fig. 6a have been removed in Figure $6 \mathrm{~b}$ by deleting the pixel regions that contain 1000 or less pixels.

After the unwanted region removal, the peak value of the liquid wave at the right wall of the tank is determined by scanning the image values, starting from the top right corner of the image and going through the bottom right corner and then going through the left, column by column.

The process mentioned above is applied to each frame to measure the peak value. Figure 7 shows the peak value of the liquid wave at the right wall of the tank versus time. Since the frame rate of the video is 29 frames per second, the scale of the horizontal axes can easily be converted to time by multiplying the frame number with $1 / 29 \mathrm{~s}$. The scale of the vertical axes shows the peak value in millimeters. Since a height of $100 \mathrm{~mm}$ corresponds to 178 pixels in our video, the resolution is $100 \mathrm{~mm} / 178$ pixels $=0.56 \mathrm{~mm}$ per pixel. Hence, the scale of the vertical axes can easily be converted to distance by multiplying the number of pixels with $0.56 \mathrm{~mm} /$ pixel.

\section{Verification and validation of measurements}

In order to verify the validity of the proposed method, the free surface displacements obtained from the video record by automated image analysis were compared with the manual measurements conducted on every 10th frame (equally spaced video frames separated by $10 / 29 \mathrm{~s}$ ) as shown in Fig. 8. The computational results did not completely match the experimental data at some frames as the frames with excessive bubbles cause some detection

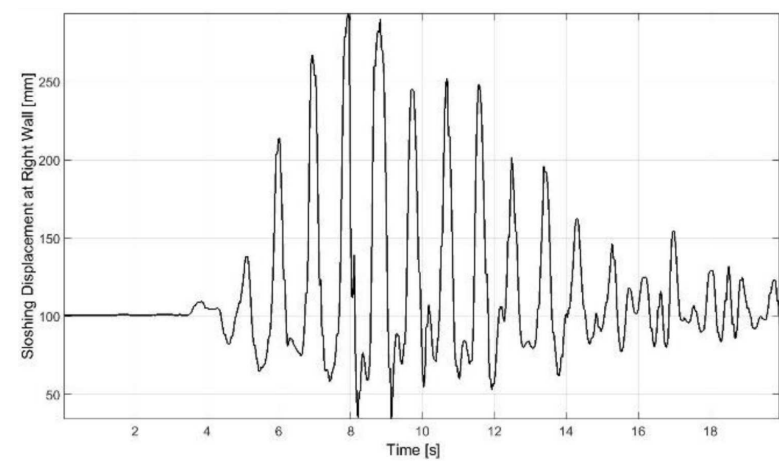

Fig. 7. Sloshing displacement at the right wall of the tank.

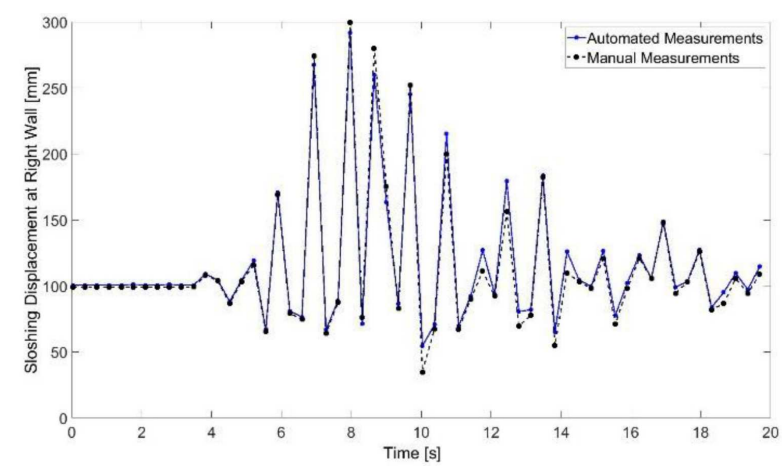

Fig. 8. Validation of the liquid level in selected frames.

errors in the peaks. Therefore, in some frames, the peak value of the liquid wave was underestimated by as much as $14.7 \%$. However, the average of the absolute errors for the selected frames was found as $4.77 \mathrm{~mm}$.

The validation study confirms that the use of the proposed measurement system with a stationary HD camera, provides a reasonable means for monitoring the sloshing displacements in transparent prismatic liquid tanks.

\section{Conclusion}

Video records are widely used in sloshing research to compare image snapshots taken at certain time instants of sloshing experiments in order to verify the free surface profiles obtained through numerical models. Considering there are thousands of frames in the video records of sloshing experiments, it is quite impractical to manually process each frame to obtain the variation of sloshing displacements at a given point throughout the experiment. Although, capacitance probes and particularly laser sensors coupled with data acquisition systems are able to measure free surface displacements with a high degree of accuracy, these systems can be quite costly and in certain cases can be invasive.

This paper proposes a non-invasive low cost method for monitoring the free surface displacements in sloshing experiments through the morphology based processing of the video records. A video acquisition-image analysis system that contains an HD camera was used to measure sloshing displacements at the right wall of the tank. 
Accuracy of the proposed measurement system depends on the spatial and temporal resolution of the video acquisition device. Since higher image resolution means more image detail, increasing the spatial resolution increases the number of pixels per centimeter. Temporal resolution affects the precision of a measurement with respect to time. Higher acquisition rates (frame rates) permit the observation of faster changing events. Hence, increase in both the spatial and temporal resolution of the video acquisition device increases the measurement accuracy.

Although, the methodology described in this paper is intended for rectangular tanks subjected to horizontal base motion, it can easily be adapted to handle other prismatic tank geometries or types of excitation such as pitch and yaw. Moreover, proposed measurement system is suitable for educational purposes and for small scale laboratories.

\section{References}

[1] P. Pal, S.K. Bhattacharyya, J. Sound Vib. 329, 4466 (2010).

[2] L.M. Hoskins, L.S. Jacobsen, B. Seismol. Soc. Am. 24, 1 (1934).

[3] E.W. Graham, A.M. Rodriguez, J. Appl. Mech. 19 381 (1952).

[4] G.W. Housner, B. Seismol. Soc. Am. 53, 381 (1963).

[5] R.A. Ibrahim, Liquid Sloshing Dynamics: Theory and Applications, Cambridge University Press, New York 2005.

[6] O.M. Faltinsen, A.N. Timokha, Sloshing, Cambridge University Press, New York 2014.
[7] M. Luo, C.G. Koh, W. Bai, Ocean Eng. 120, 52 (2016).

[8] S.Y. Kim, K.H. Kim, Y. Kim, Ocean Eng. 94, 199 (2015).

[9] J. Mei-Rong, R. Bing, W. Guo-Yu, W. Yong-Xue, J. Hydrodyn. 26, 751 (2014).

[10] F. Pistani, K. Thiagarajan, Ocean. Eng. 52, 60 (2012).

[11] A. Souto-Iglesias, E. Botia-Vera, A. Martin, F. PerezArribas, Ocean. Eng. 38, 1823 (2011).

[12] E. Frosina, A. Senatore, A. Andreozzi, F. Fortunato, P. Giliberti, Energies 11, 682 (2018).

[13] U. Tosun, R. Aghazadeh, C. Sert, M.B. Özer, Exp Therm. Fluid Sci. 88, 423 (2017).

[14] T.D. Ancheta, R.B. Darragh, J.P. Stewart, E. Seyhan, W.J. Silva, B.S.J. Chiou, K.E. Wooddell, R.W. Graves, A.R. Kottke, D.M. Boore, T. Kishida, J.L. Donahue, PEER NGA-West2 Database, PEER 2013/03, Pacific Earthquake Eng. Research Center, University of California, Berkeley 2003.

[15] Recommendation ITU-R BT.601-7, Studio encoding parameters of digital television for standard 4:3 and wide screen 16:9 aspect ratios, ITU-R, Geneva 2017.

[16] R.C. Gonzalez, R.E. Woods, Digital Image Processing, 4th ed., Pearson, 2017.

[17] M. Sonka, V. Hlavac, R. Boyle, Image Processing, Analysis and Machine Vision, 3rd ed., Thomson Learning, 2008.

[18] R.M. Haralick, L.G. Shapiro, Computer and Robot Vision, Vol. I, Addison-Wesley, Boston (MA) 1992.

[19] J. Serra, Image Analysis and Mathematical Morphology, Academic Press, Orlando (FL) 1982.

[20] P. Maragos, R. Schafer, Proc. IEEE 78, 690 (1990). 